\title{
3D GPR data acquisition and the influence of positioning errors on image quality
}

\section{Conference Paper}

\section{Author(s):}

Kruk, Jan van der; Groenenboom, J.; Zeeman, J.H.

Publication date:

2001

Permanent link:

https://doi.org/10.3929/ethz-a-004364119

Rights / license:

In Copyright - Non-Commercial Use Permitted 


\title{
3D GPR data acquisition and the influence of positioning errors on image quality
}

\author{
J. Groenenboom, J. van der Kruk* and J.H. Zeeman, \\ Extended Abstract EAGE \\ 63rd Conference and Technical Exhibition, Amsterdam, \\ 11-15 June 2001, 4 pp.
}

Delft University of Technology, Faculty of Applied Earth Sciences, Section of Applied Geophysics

*Now working at:

Institute of geophysics, Swiss Federal Institute of Technology ETH-Hoenggerberg, CH-8093, Zurich, Switzerland 



\title{
P141 3D GPR DATA ACQUISITION AND THE INFLUENCE OF POSITIONING ERRORS ON IMAGE QUALITY
}

J. GROENENBOOM, J. VAN DER KRUK and J.H. ZEEMAN

Delft University of Technology, Applied Earth Sciences, Mijnbouwstraat 120, 2628 RX Delft, The Netherlands

\begin{abstract}
Summary
Two 3D GPR data sets have been acquired over the same test site. The first data set was recorded manually. Positioning errors caused discontinuous behaviour of the measurements along the crossline direction. After application of a migration algorithm we observe that the image quality is distorted. The second data set was acquired with an automated measurement frame with high positioning accuracy. The data obtained has better crossline continuity and the final image quality is significantly improved. For GPR applications that need high resolution images, like landmine detection, we learn that positioning of the radar system is crucial. The high quality data set can be used to assess the performance of various three-dimensional image algorithms.
\end{abstract}

\section{Introduction}

Ground penetrating radar has become an established technology for shallow subsurface characterisation. Successful results have been obtained for applications such as groundwater investigations and road inspection using data acquisition along lines. Some applications such as utility detection or landmine detection (Groenenboom and Yarovoy, 2000) require three-dimensional data acquisition, because the subsurface targets are not necessarily found along a single line. To obtain unaliased 3D subsurface images the spatial sampling must be sufficiently small both in the inline and crossline direction. As a consequence, threedimensional manual GPR data acquisition is time-consuming, labour-intensive and most of all prone to positioning errors. Methods are being developed for accurate positioning information (see e.g. Lehmann and Green, 1999). At the same time, new three-dimensional imaging algorithms for GPR data are being developed (Kruk et al, 2001; Wang and Oristaglio, 2000, Kruk et al., 2000). To assess the performance of the various algorithms we need a three-dimensional data set of high quality, for which the acquisition parameters are in tune with the requirements of the imaging algorithm. Modeling a full three-dimenionsal GPR survey with a realistic subsurface configuration is a huge computational task. Therefore we decided to measure a three-dimensional data set on a controlled testsite containing a sandpit with rather homogeneous soil conditions. Here, we will describe our experience with three-dimensional GPR data acquisition and some preliminary conclusions on imaging results.

\section{Manual data acquisition}

In the spring of 1999 we carried out a three-dimensional GPR survey in the testsite. A broadside $900 \mathrm{MHz}$ PulseEkko antenna configuration was used and the radar system was manually moved with steps of $5 \mathrm{~cm}$. The distance between lines was $5 \mathrm{~cm}$ as well. In the sandpite two metal pipes were buried in addition to some metal and plastic spheres. In Figure 1 on the left we show a line of data. Two clear hyperbolic events are present related to the metal pipes. By selecting the same scan number for each line we obtain a virtual line of data along the crossline direction. This is shown in Figure 1 on the right. The continuity of the reflected event is not as smooth as the hyperbolic event in the inline direction. We proposed that this discontinuity was caused by positioning errors related to the fact that the data were measured manually with a string odometer for each line, whereas feathering of the GPR system in the crossline direction was still possible. In Figure 2 we illustrate the GPR equivalent to feathering. 

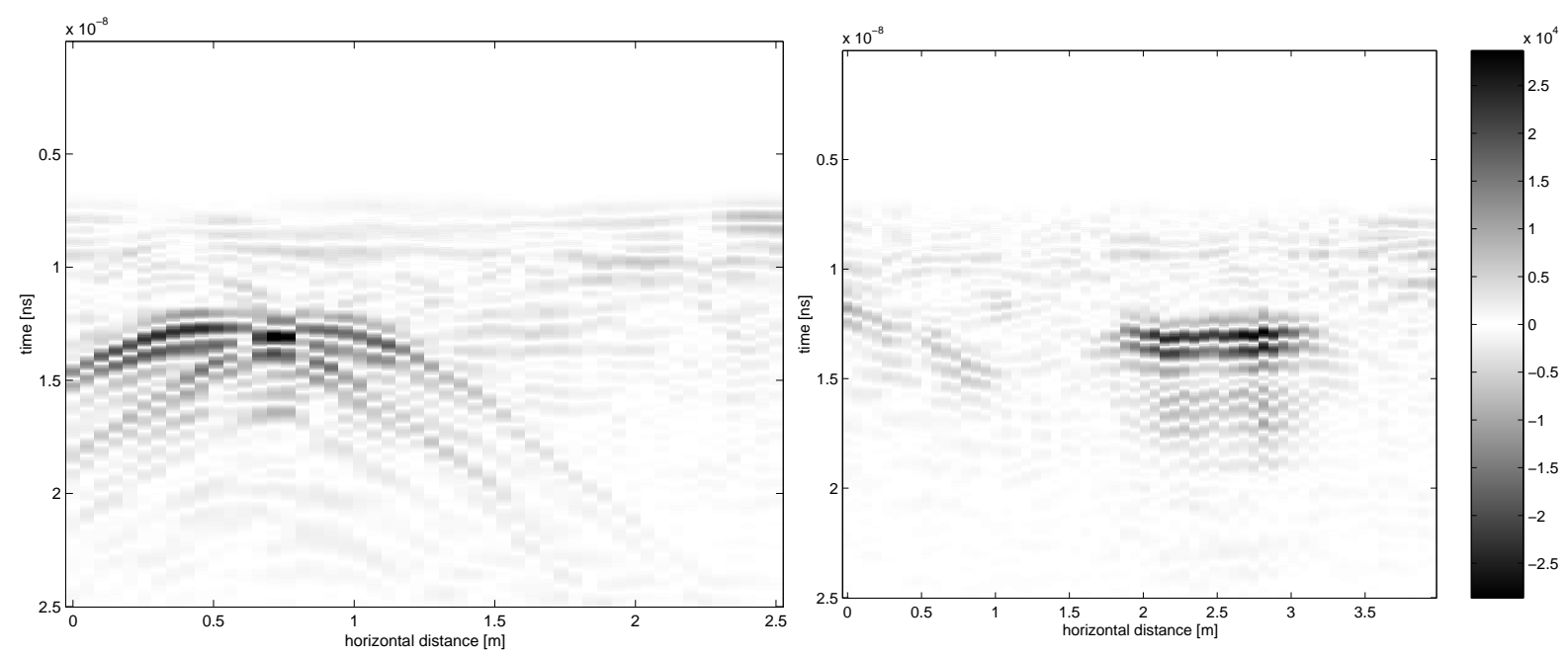

Figure 1: The data measured manually along the inline direction (left) and the crossline (right) direction. Note the discontinuities of the reflection of the pipe on the right.

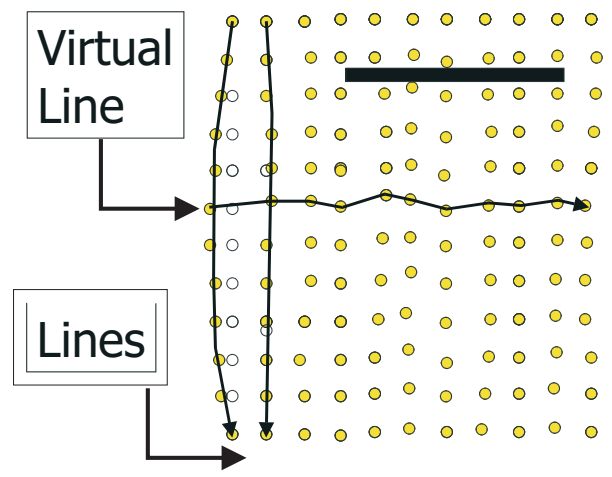

Figure 2: The positioning errors due to feathering of the GPR system, which will become more apparent in the crossline continuity of the data

\section{Automated data acquisition}

To verify the postulate that the image quality was distorted due to positioning errors, we decided to design a measurement frame for automated data acquisition. The automated data acquisition frame, see Figure 2 has an effective measurement size of 3 by 3 meter and in principle a complete $\mathrm{C}$-scan can be measured without human interference. The positioning error is less than a millimeter. With the new measurement frame we measured a three-dimensional data set over the same area, now with a spacing of $2.5 \mathrm{~cm}$ in the inline and crossline direction. The data are shown in Figure 4. We observe that the continuity along the crossline has severely improved

\section{Imaging results}

To show that the measurement frame can improve image quality we selected a subset of the data acquired with the measurement frame with a spatial interval of 5 centimeters for a fair comparison with the manually acquired data. On both data sets we applied standard Gazdag migration. In Figure 5 we show slices of image results at the depth levels of the two straight pipes. We observe that the images of the pipes are curved whereas the pipes are straight with the automatically acquired data. Hence the image distortion of the two straight pipes is likely caused by positioning errors. Finally we applied Gazdag migration to the full data set with an inline and crossline sampling interval of 2.5 centimeters, resulting in Figure 6. Here, we observe that the resolution of the images has substantially improved. Another obvious advantage of the 


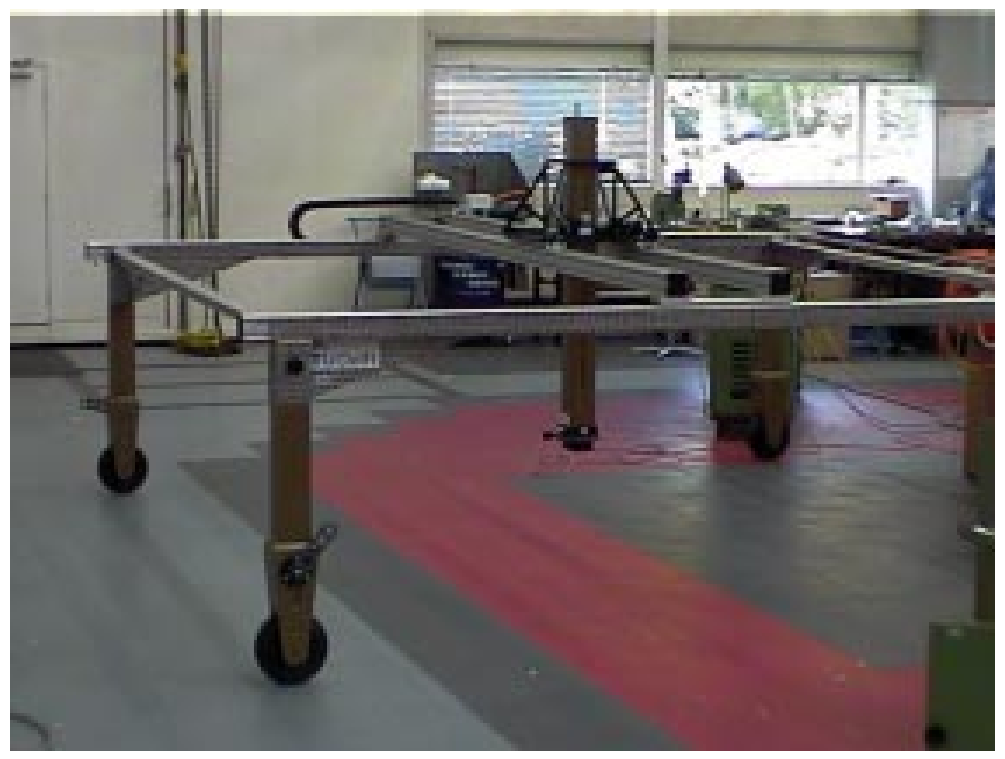

Figure 3: The measurement frame in the laboratory, with an effective measurement size of 3 by 3 meter. Note the possibility to measure with elevated antennas as well as ground coupled antennas.

measurement frame is to acquire the data with small spatial interval. This data set would be very labour intensive to acquire manually.

\section{Conclusions}

Currently, quite some GPR research focuses at designing imaging algorithms for obtaining high-quality three-dimensional images. For example, landmine detection requires crossline image resolution in the order of centimeters. Our experience with three-dimensional GPR data acquisition shows that accurate positioning of the GPR system then becomes crucial. With the measurement frame designed to obtain high quality data we have now available a data set which can be used to assess the performance of various three-dimensional imaging algorithms.

\section{Acknowledgments}

This research is supported by the Dutch Technology Foundation (STW).

\section{References}

[1] Groenenboom, J. and Yarovoy, A.G. (2000) Data processing for a Landmine detection dedicated GPR, GPR2000, Proc. of the Eight International Conference on Ground Penetrating Radar, May 2000.

[2] Lehmann, F. and Green, A.G. (1999) Semiautomated georadar data acquisition in three dimensions, Geophysics 64, pp 719-731.

[3] van der Kruk, J, Wapenaar C.P.A and Fokkema, J.T. Comparison of resolution functions of 3-D multi-component with 3-D single-component imaging algorithms for ground penetrating radar data, Proceedings 5th SEGJ International Symposium - Imaging Technology - The Society of Exploration Geophysicists of Japan (SEGJ), Tokyo, Japan, Januari 24-26, 2001.

[4] Wang, T. and Oristaglio, M.L. GPR imaging using the generalized Radon transform. Geophysics 65, 52000. pp1553-1559.

[5] van der Kruk, J, Wapenaar C.P.A and Fokkema, J.T. Multi-component 3-D Imaging of ground penetrating radar data using matrix inversion in the spatial Fourier domain, Proceedings Eight international conference on GroundPenetrating Radar, Queensland, Australia, May 23-26, 2000, pp. 508-513. 

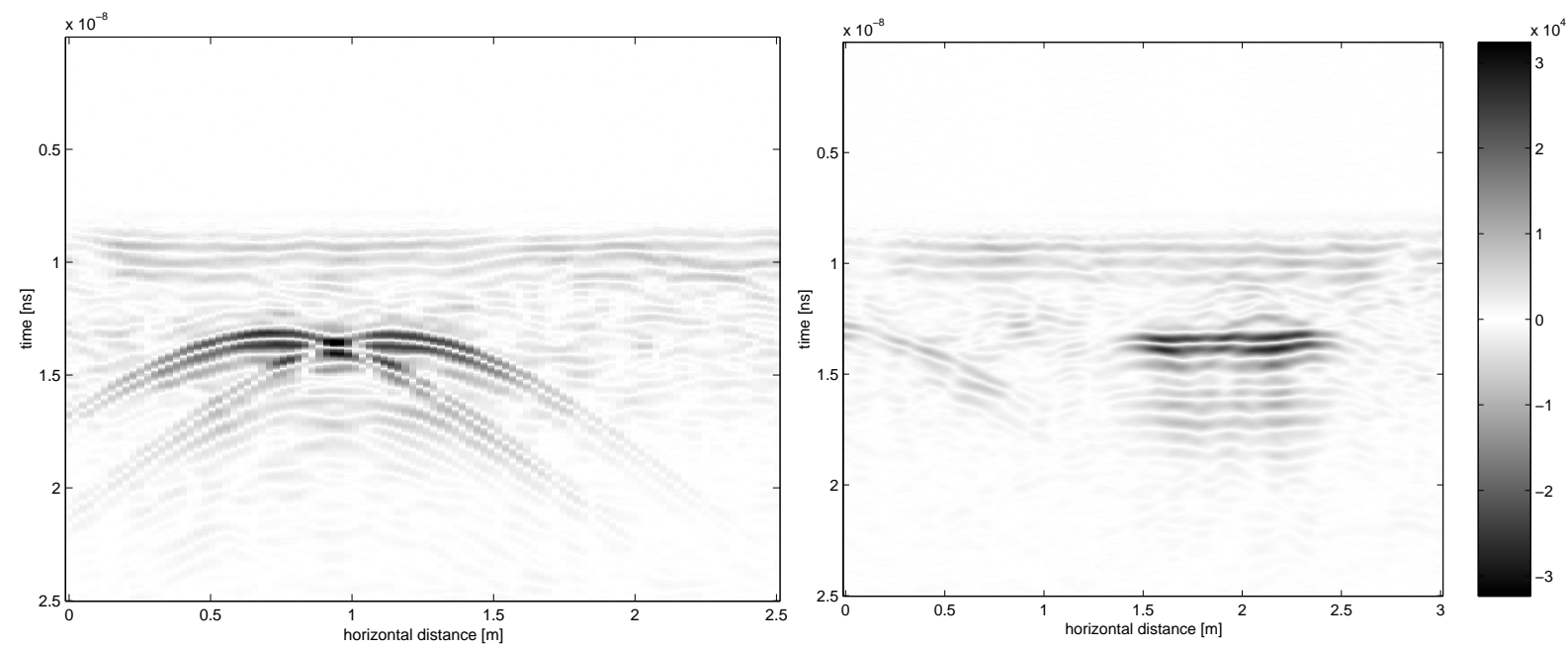

Figure 4: The new data along the inline (left) and crossline (right) direction. Note the continuity of the pipe reflection in the crossline direction.
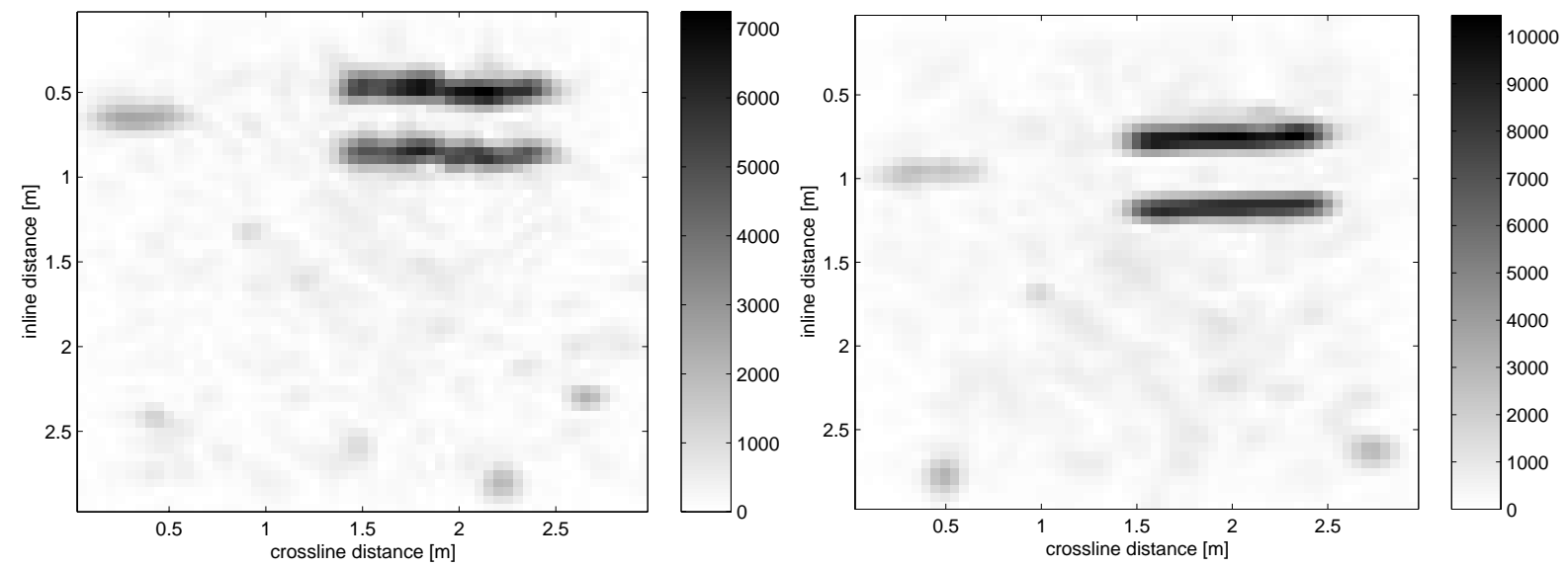

Figure 5: The imaging results for the manually acquired data on the left, and the automatically acquired data on the right, both with a spatial interval of 5 centimeters.

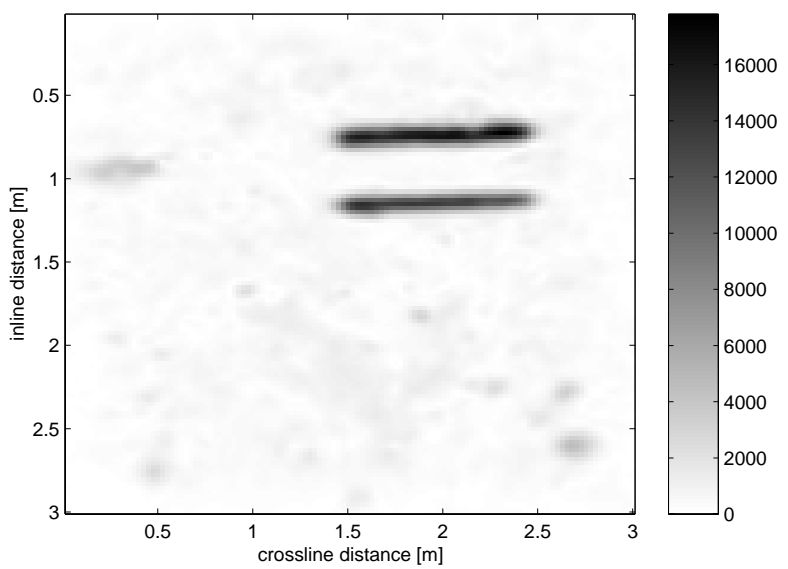

Figure 6: The imaging results of the data measured with the acquisition frame with a spatial interval of 2.5 centimeter. 